\title{
Study Evaluation of Image inpainting based on S-R algorithm
}

\author{
Nitin P. Jagtap ${ }^{1}$, Vishal S. Dusane ${ }^{2}$, Rohini P. Pawar ${ }^{3}$, Nitin B. Patil ${ }^{4}$ \\ Department of Information Technology, Shram Sadhna Bombay Trust COET, Jalgaon, India ${ }^{1,2,3,4}$
}

\begin{abstract}
Image inpaiting is the process of reconstructing or modifying the deteriorated or damage part of the image, just like in world of museum or valuable paintings the process of the inpainting is done by skill expert conservator. This inpainting process is focuses on to reduce the computational complexity of image structures. In this paper we introduced two algorithms that is diffusion based and super resolution algorithm. By comparing two algorithms we concluded that the super resolution algorithm is more superior than the diffusion based algorithm in the cases of clarity and time. In one survey, the innovative Digital photography is changing the way we communicate, socialize, and document events around us but they face some problem like blur image or some damaged part in the valuable image so it is need some new application software that help to solve these problems hence in this paper we introduced SR algorithms.
\end{abstract}

Keywords: Image inpainting, Blur image, Diffusion based algorithm, SuperResolution algorithm.

\section{INTRODUCTION}

The given input image is first in painted by a nonparametric patch sampling. Compared to existing approaches, some improvements have been done. The in painted of a coarse version of the input image is focuses on to reduce the computational complexity, to be less close to noise and to work with the powerful calculation of image structures[1]. From the low-resolution in painted image, a single- image super-resolution is applied to recover the details of missing areas. Experimental results on natural images and texture synthesis demonstrate the effectiveness of the proposed method [1].

The method of inpainting is derived from the ancient art of restorting the damage image by expert image restorer's in art gallery. The image inpaiting follows this ancient process and this algorithm uses this ancient process and perform inpaiting automatically, so the author use the S-R algorithm and this algorithm uses this ancient techniques in a way that it looks reasonable to the viewer. By using this technique, the selected region from the image is removed completely but the region we have to be removed cannot be recovered by any mathematical method. Therefore the goal for image inpaiting is not to recover the original image, but it creates new one image which is resemblance with original image. Such software application has several benefits one use in reconstructing the damaged part of the images, year ago or ancient, people were preserve their important visual work.

In Image Inpainting sometimes there is some part may be blurish or incomplete so, to filled out that incomplete region can be filled using method called as image inpainting to filled out that image first it can check it's all pixels and that pixel can be calculated by $16 * 16$ and they can also checks its RGB value according to that RGB value the incorrect part may be compare with that pixel And match that pixel and its nearest neighbour's .it can also check its computational speed and the input image is stored in dictionary that can be used in filled the image part.

Copyright to IJARCCE

DOI 10.17148/IJARCCE.2015.4234

\section{LITERATURE SURVEY}

Olivier Le eur et al. proposed a structure which combines inpainting and super-resolution. Intially, a low-resolution image is built from the original picture [1]. Yunqiang Liu et al. proposed a reformulation of the examplar-based approach as a discrete global optimization problem. The proposed energy functional is written in terms of the offset map (assigning to each point of the inpainting domain a corresponding point in the known part of the image). The energy term consists data attachment term to promise the continuity of the reconstruction at the boundary of the inpainting domain and a term that favours a spatially unity in the image completion [2]. R. Mart'mez-Noriega et al. proposed an improvement to the priority order in addition with an edge detection algorithm. To solve the global inpainting optimization problem a scheduling is proposed that requires the computation of many distances at the initialization. For each patch located at the boundary between the known and the unknown region, the distance between this patch and all known patches are computed [3].

\section{A. Diffusion Based Algorithm and Examplar Based Image Inpaiting Algorithm}

Existing method categorized into two approaches first approach is diffusion based algorithm and second one is exemplar based algorithm. In diffusion based it can be matches the pixel line by line or linear structure or in isophates direction. Unfortunately, as a result the diffusion based method gives some blur or unclear image, when the region for inpainting is so large. It required more time to inpaint an image.

The examplar-based method follows two classical steps as follows:

- $\quad$ Filling Order computation

- Texture synthesis

While in examplar based method, the filling order computation defines a measure of priority for each patch in order to distinguish the structures from the textures. The

Copyight to IJARCCE

155 
priority of a patch centered on $\mathrm{p}$ is just given by a data term. It cans match the best pixel from known image. Theresult in an approximation of the missing region. It require less time to inpaint an image as compare to diffusion based algorithm. Three different data terms have been tested [1].

- $\quad$ gradient-based priority

- tensor-based

- $\quad$ sparsity-based

At the time of inpainting, a pixel matching of the image is performed between the current patch $\mathrm{V}$ and neighbouring patches $\mathrm{k}$, ka that belong to the known part of the image. By using a non- local means approach a similarly weight $\mathrm{Va}, \mathrm{Kb}$ is computed for each pair of patches. The sparsity term is defined as:

$$
V a, K b=\exp \left(-\frac{s(\varphi a, \varphi a, a b}{h}\right)
$$

There are two major operations first one is non-parametric approach and second is inpainting. In non-parametric approach patch sampling method used to fill-in missing regions. However, rather than filling in missing regions at the original resolution, and in the inpainting method is easy to perform as compare to non-parametric approach. If the image size smaller than it can reduces its computational time the super resolution image inpainting consist of mainly two components.

- Inpainting

- Super Resolution algorithm.

In super resolution based image inpainting it consists of following steps:

- It can build a low resolution image apart from original picture.

- The resolution algorithm then use to fill the low resolution image.

- To improve the quality of low resolution inpainted region the super resolution algorithm is used.

\section{III.FRAMEWORK}

As shown in the figure first we have to select appropriate region for image inpainting then the particular image is converted into low resolution image. After the selection of particular region the super resolution algorithm is applied on that particular region. As a result, this well increases the quality of the image.

The samples are taken to clear the concept of the image inpainting an image taken which may consist of damage in the image and then the whole image can be stored as input after that the damage part can be selected and on that damage part the super resolution algorithm is applied. After completing all the process we have to get a clear image that image can be generated as an output shown in above figure. 
- No 5 Indicates that, stitching it means that according to SRAlgorithm it can check the RGB value and according to RGB value it can decide the priority values and then it places highest priority pixel to selected region.

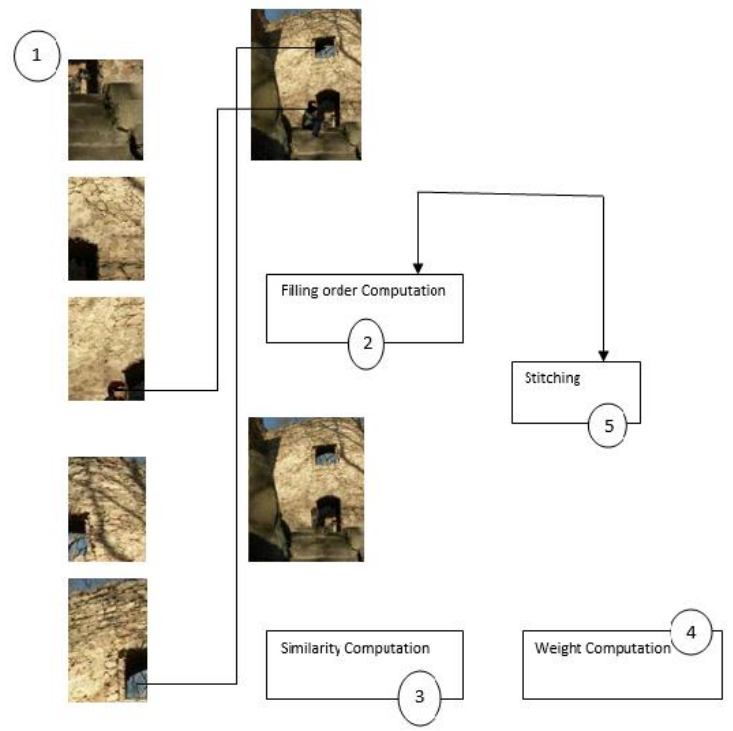

Fig 1. Flowchart for S-R Algorithm

\section{IV.CONCLUSION}

In this paper we studied new algorithm for image inpainting that attempts to import the basic techniques use by professional restorators in ancient time. In proposed algorithm the basic idea behind the S-R algorithm but in modify version to transform the information from surrounding area in isophates direction. In this application the user needs to select region or particular frame for image inpainting the operation is performed automatically within a minute. The output images are sharp and clear with higher magnification factor and decreases computational complexity.

Firstly, Resercher will take sequence of damage images, will find new algorithm which will deal on sequence of damage images and which will the advance version of S-R algorithm. Second, Researcher will do the above proposed system by using artificial intelligent as par as time concern.

\section{REFERENCES}

[1] Olivier Le Meur and Christine Guillemot,"Super-Resolution-based Inpainting" University of Rennes 1, France; INRIA Rennes, France olemeur@irisa.fr, christine.guillemot@inria.fr.2013

[2] R. Martinez-Noriega, A. Roumy, and G. Blanchard, "Exemplarbased image inpainting: Fast priority and co- Herent nearest neighbor search," in Machine Learning for Signal Processing (MLSP), 2012 IEEE International Workshop on, Sept 2012, pp. 1-6.

[3] A. Criminisi, P. P'erez, and K. Toyama, "Region filling and object removal by exemplar-based image inpainting," IEEE Trans. Im. Proc., vol. 13, no. 9, pp. 1200-1212, Sept. 2004.

[4] O. Le Meur, J. Gautier, and C. Guillemot, "Examplar-based inpainting based on local geometry," in Proc. 18th IEEE Int. Conf. Image Process., Sep. 2011, pp. 3401-3404.

[5] C. Barnes, E. Shechtman, A. Finkelstein, and D. B. Goldman, "Patch- Match: A randomized correspondence algorithm for structural image editing," ACM Trans. Graph., vol. 28, no. 3, p. 24, Aug. 2009.

[6] Efros, A.A., Leung, T.K.: Texture synthesis by non-parametric sampling. In: International Conference on Computer Vision. (1999) 1033-1038

[7] Chang, H., Yeung, D.Y., Xiong, Y.: Super-resolution through neighbor embedding. In: Computer Vision and Pattern Recognition. Volume I. (2004) 275-282 